\title{
Upregulation of Erythropoietin and its Receptor Expression in the Rat Carotid Body during Chronic and Intermittent Hypoxia
}

\author{
S.Y. LAM, G.L. TIPOE ${ }^{1}$, AND M.L. FUNG \\ Departments of Physiology and ${ }^{1}$ Anatomy, The University of Hong Kong, Faculty of \\ Medicine Building, 21 Sassoon Road, Pokfulam, Hong Kong SAR, China
}

\begin{abstract}
The carotid body (CB) plays important roles in cardiorespiratory changes in intermittent hypoxia (IH). Erythropoietin (EPO), a hypoxiainducible factor (HIF)-1 target gene, is present in the chemoreceptive type-I cells in the CB but its expression and role in IH resembling sleep apnoeic conditions are not known. We hypothesized that IH upregulates the expression of EPO and its receptor (EPOr) in the rat $\mathrm{CB}$. The $\mathrm{CB}$ expressions of EPO and EPOr were examined in rats breathing $10 \% \mathrm{O}_{2}$ (in isobaric chamber for $\mathrm{CH}, 24$ hour/day) or in $\mathrm{IH}$ (cyclic between air and $5 \% \mathrm{O}_{2}$ per minute, 8 hour/day) for 3-28 days. Immunohistochemical studies revealed that the EPO and EPOr proteins were localized in CB glomic clusters. The proportional amount of cells with positive staining of EPO immunoreactivities was significantly increased in both $\mathrm{IH}$ and $\mathrm{CH}$ groups when compared with the normoxic control. The EPO expression was more markedly increased in the $\mathrm{CH}$ than that of the $\mathrm{IH}$ groups throughout the time course, reaching a peak level at day 14. The positive EPOr immunostaining was increased significantly in the 3-day $\mathrm{CH}$ group. By day 14, the EPOr expression elevated considerably at peak levels in both $\mathrm{IH}$ and $\mathrm{CH}$ rats, whereas the elevation was greater in the $\mathrm{CH}$ rats. These results suggest an upregulation of EPO and its receptor expression in the rat $\mathrm{CB}$ under $\mathrm{IH}$ and $\mathrm{CH}$ conditions, presumably mediated by the activation of HIF-1 pathway. The increased EPO binding to its receptor might play a role in the enhancement of $\mathrm{CB}$ excitability during the early pathogenesis in patients with sleepdisordered breathing.
\end{abstract}

\section{INTRODUCTION}

Carotid body (CB) plays important roles in cardiorespiratory changes in both chronic hypoxia $(\mathrm{CH})$ and intermittent hypoxia (IH) (Gonzalez et al. 1994; Prabhakar 2001). During CH, the hypertrophy and hyperplasia of $\mathrm{CB}$ glomus cells contribute to the $\mathrm{CB}$ enlargement as observed in high altitude dwellings and patients with chronic obstructive 
pulmonary disease (Bee and Howard 1993; Heath et al. 1970). Under IH conditions, hypoxic chemosensitivity and the ventilatory chemoreflex were significantly augmented in the rat CB (Peng et al. 2004). Also, IH enhances the hypoxia-evoked neurotransmitter release from the $\mathrm{CB}$ as well as PC12 cells (Kim et al. 2004).

Hypoxia-inducible factor (HIF)-1 $\alpha$ plays a physiological role in $\mathrm{CH}$ and $\mathrm{IH}$. The expression of HIF- $1 \alpha$ and the transcriptional regulation of an array of HIF-regulated genes in the rat $\mathrm{CB}$ is an essential molecular mechanism for the structural remodelling and functional changes of the organ under hypoxic conditions (Lam et al. 2008; Prabhakar 2001). Upregulation of the HIF-regulated genes such as vascular endothelial growth factor (VEGF), VEGF receptors, endothelin-1 and tyrosine hydroxylase are crucial for the CB response to hypoxic conditions (Chen et al. 2002; Fung 2003; Lam et al. 2008; Tipoe and Fung 2003).

Erythropoietin (EPO), a HIF-regulated gene, despite its novel erythropoietic function in enhancing red blood cells production, has been shown to modulate the ventilatory response to reduced oxygen supply mediated by activation of $\mathrm{CB}$ chemoreceptors. In fact, the $\mathrm{CB}$ was proposed to be an endocrine gland participating in the control of erythropoiesis (Tramezzani et al. 1971). Recent study has demonstrated that exogenous EPO could activate the $\mathrm{CB}$ chemoreceptor during $\mathrm{CH}$. In addition, EPO receptor (EPOr) was found to be present in the chemoreceptive type-I cells in the CB (Soliz et al. 2005). Furthermore, high EPO plasma levels could modulate the CB chemotransduction in $\mathrm{CH}$ (Soliz et al. 2007). However, the expression and role of EPO under IH resembling sleep apnoeic conditions are unclear. It is hypothesized that the expression of EPO and its receptor (EPOr) in the rat CB could be regulated by $\mathrm{CH}$ or $\mathrm{IH}$. The aim of the present study was to examine the $\mathrm{CB}$ expressions of EPO and EPOr in rats breathing $10 \% \mathrm{O}_{2}$ for $\mathrm{CH}$ or in cyclic $\mathrm{O}_{2}$ levels between 5-21\% for IH for 3-28 days. Our results suggest an upregulation of EPO and its receptor expression in the rat $\mathrm{CB}$ under $\mathrm{IH}$ and $\mathrm{CH}$ conditions, presumably mediated by the activation of HIF-1 pathway.

\section{METHODS}

The experimental protocol for this study was approved by the Committee on the Use of Live Animals in Teaching and Research of The University of Hong Kong. Male Sprague-Dawley rats aged 28 days were randomly divided into groups for the treatment of $\mathrm{CH}$ or $\mathrm{IH}$ and for normoxic $(\mathrm{Nx})$. While the $\mathrm{Nx}$ controls were kept in room air with the same housing and maintenance, both $\mathrm{CH}$ and $\mathrm{IH}$ rats were kept in acrylic chambers for normobaric hypoxia and had free access to water and chow (Tipoe and Fung 2003). The oxygen fraction inside the chamber was kept at $10 \pm 0.5 \%, 24 \mathrm{~h}$ per day for the $\mathrm{CH}$ group and was cyclic from 
21 to $5 \pm 0.5 \%$ per min, 60 cycles / h, $8 \mathrm{~h}$ per day diurnally for the IH group in which the inspired oxygen level fell to $4-5 \%$ (nadir arterial oxygen saturation $c a .70 \%$ ) for about $15 \mathrm{sec}$ per min, which mimicks the recurrent episodic hypoxemia in patients with obstructive sleep apnoea (Fletcher 2001). The desired oxygen level was established by a mixture of room air and nitrogen that was regulated and monitored by an oxygen analyzer (Vacumetrics Inc., CA, USA). Carbon dioxide was absorbed by soda lime granules and excess humidity was removed by a desiccator. The chamber was opened twice a week for an hour to clean the cages and replenish food and water. The rats were exposed to hypoxia for 3, 14 and 28 days and were immediately used in experiments after taken out of the chambers.

Following deep anesthesia with halothane, the rat was decapitated and the carotid bifurcation was excised rapidly. The CB was carefully dissected free from the bifurcation and was fixed in neutral buffered formalin for 72 hour. Tissues were processed routinely for histology and embedded in paraffin blocks. Serial sections of $5 \mu \mathrm{m}$ thickness were cut and mounted on silanized slides (DAKO, Denmark). Sections were kept in the oven overnight at $56^{\circ} \mathrm{C}$. Consequently, sections were deparaffinized, rehydrated and immunostained with antiserum to the following proteins: EPO (rabbit IgG antibody, 1:500 dilution, Cat \# E0271, Sigma, USA); EPOr (goat IgG antibody, 1:500 dilution, Cat \# E4644, Sigma, USA), using LSAB kit (K0690, DAKO). In brief, sections were immersed in antigen retrieval solution $(0.1 \mathrm{M}$ citric acid buffer, $\mathrm{pH} 6.0$ ) for $10 \mathrm{~min}$ at $98^{\circ} \mathrm{C}$. To block endogenous peroxidase activity, the sections were immersed in 3\% hydrogen peroxide for $5 \mathrm{~min}$ at room temperature. All sections were immersed in a solution containing $0.01 \%$ trypsin and calcium chloride for $5 \mathrm{~min}$ at room temperature and were pre-incubated with $20 \%$ normal serum for 2 hour to reduced non-specific binding for the antiserum. Then sections were incubated with the corresponding primary antibodies in $0.05 \mathrm{M}$ Tris- $\mathrm{HCl}$ buffer, respectively, containing $2 \%$ bovine serum albumin overnight at $4^{\circ} \mathrm{C}$. Sections were washed three times in PBS, and then incubated with biotinylated link agent and streptavidin peroxidase for 30 $\min$ at room temperature. Finally, sections were washed and the peroxidase was visualized by immersing in $0.05 \%$ diaminobenzidine containing $0.03 \% \mathrm{H}_{2} \mathrm{O}_{2}$ in Tris- $\mathrm{HCl}$ buffer $(\mathrm{pH} 7.5$ ). Sections were counterstained mildly with hematoxylin. Control sections were prepared by substitution of primary antibodies with buffer or normal serum, and liquid phase pre-adsorption of the used antibodies with excess blocking peptides (Sigma, USA).

The immunoreactivities of EPO and EPOr were measured using the Leica QWIN Imager Analyzer (Cambridge, UK). Immunostained sections were captured with a CCD JVC camera using a Zeiss Axiophot microscope at $100 \mathrm{X}$ objective. The luminance incident light passing 
through each section was calibrated using the set-up menu where the grey pixel values were set to 0 and 1.00 . Once the setup was done, five fields per section from one $\mathrm{CB}$ of one animal were measured. The percent area of positive stain for both EPO and EPOr protein was measured by detecting the positive brown cytoplasmic stain divided by the sum areas of the reference field. A total of 20 fields for $4 \mathrm{CBs}$ from four different animals at each time-point namely day 3, 14 and 28 were determined. The mean value of the 20 fields was calculated to represent each time-point.

GraphPad Prism ${ }^{\circledR}$ software (GraphPad Software, Inc., San Diego, USA) was used to analyze the data. A non-parametric Mann-Whitney $U$-test was used to compare differences between time-points. A $p$-value $<0.05$ was considered statistically significant.

\section{RESULTS}

Immunohistochemical studies revealed that the EPO and EPOr proteins were positively stained in most of cells throughout the CBs of rats exposed to $\mathrm{CH}$ and $\mathrm{IH}$ treatment $(n=5)$. The positive staining of EPO and EPOr were localized in CB glomic clusters (Figs 1A and 2A). Moreover, the immunostaining was not observed in the $\mathrm{CB}$ of the corresponding control sections incubated with normal serum instead of the primary antibodies (data not shown).

The proportional amount of cells with positive staining of EPO immunoreactivities (IR) was significantly increased in both $\mathrm{IH}$ and $\mathrm{CH}$ groups when compared with the Nx control. The EPO expression was markedly increased in the 3-day group of both $\mathrm{IH}$ and $\mathrm{CH}$ rats, while the elevation was less in $\mathrm{IH}$ than $\mathrm{CH}$. A sustained significant increase of EPO expression was found throughout the time course and reached a peak level at day 14 in both $\mathrm{IH}$ and $\mathrm{CH}$ rats, but augmented in a greater extent in $\mathrm{CH}$ than $\mathrm{IH}$ (Fig. 1B).

The positive staining of EPOr expression was markedly increased in the 3-day groups of $\mathrm{CH}$ rats. By day 14, the EPOr expression elevated considerably and reached a peak level in both the IH

and $\mathrm{CH}$ rats, whereas the elevation was greater in $\mathrm{CH}$ than $\mathrm{IH}$. Moreover, EPOr expression reduced to levels above the $\mathrm{Nx}$ control in the $\mathrm{CH}$ group but remained elevated in IH by day 28 (Fig. 2B).

As reported previously, positive staining of HIF- $1 \alpha$ in the rat CB is significantly more in the $\mathrm{CH}$, but not in the IH group (Fig. 3 modified from (Lam et al. 2008)). Collectively, these results suggest an upregulation of the expression of EPO and its receptor in the rat $\mathrm{CB}$ under $\mathrm{IH}$ and $\mathrm{CH}$ conditions, presumably mediated by the activation of HIF-1 pathway. These observations are consistent with the hypothesis that EPO plays a functional role in the enhancement of CB excitability under hypoxic conditions. 


\section{DISCUSSION}

EPO acts as a key regulator of erythropoiesis, by promoting the survival and proliferation of erythroid precursor cells (Jelkmann 1992). The kidney produces EPO in response to hypoxia, and thereby increases the number of red blood cells in order to increase the tissue oxygen supply (Pagel et al. 1992). Evidence suggest that the CB is an endocrine gland playing a part in the control of erythropoiesis despite its classical chemoreceptive function (Tramezzani et al. 1971). The present study demonstrated that (1) EPO and its receptors, EPOr were expressed and localized in CB glomic clusters; (2) The proportional amount of cells with positive staining of EPO and EPOr immunoreactivities were significantly increased in both $\mathrm{IH}$ and $\mathrm{CH}$ groups when compared with the Nx control. These findings unequivocally support the hypothesis that $\mathrm{CH}$ and $\mathrm{IH}$ upregulate the EPO and its receptor expression in the rat $\mathrm{CB}$.

A

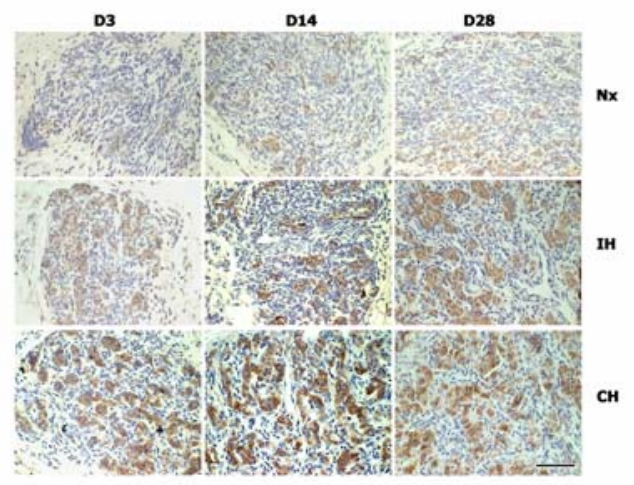

B

EPO Protein Expressions

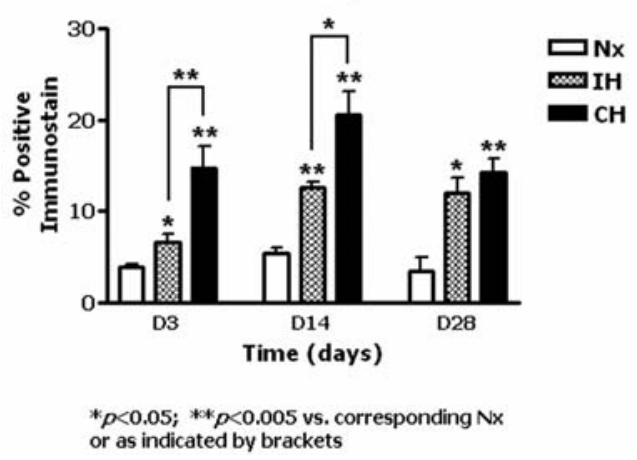

Figure 1. A: Immunohistochemical localization of EPO in 3-, 14-, and 28-day treatment of $\mathrm{IH}, \mathrm{CH}$ and their corresponding $\mathrm{Nx}$ in rat $\mathrm{CB}$. Bar $=40 \mu \mathrm{m}$. B. Protein expressions of EPO in carotid body of rat in Nx, IH and $\mathrm{CH}$ for 3 (D3), 14 (D14) and 28 days (D28). Data are presented in \% area with positive staining of EPO immunoreactivity and are expressed as mean $\pm \mathrm{SEM}$ ( $\mathrm{n}=5$ for each group). 
A

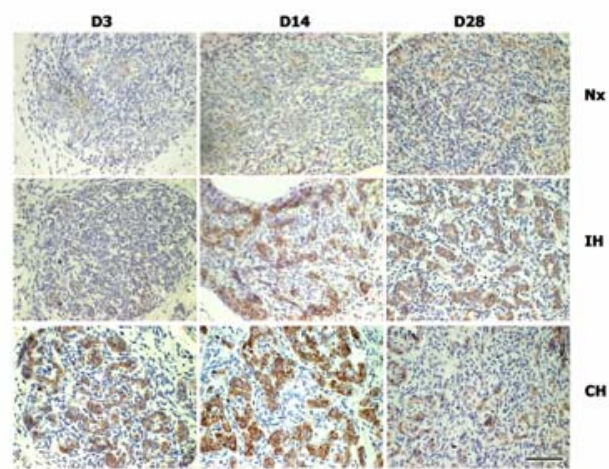

B

EPOr Protein Expressions

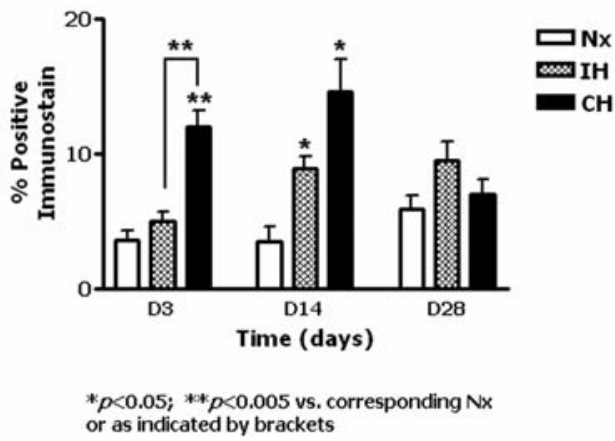

Figure 2. A: Immunohistochemical localization of EPOr in 3-, 14- and 28-day treatment of $\mathrm{IH}, \mathrm{CH}$ and their corresponding $\mathrm{Nx}$ in rat $\mathrm{CB}$. Bar $=40 \mu \mathrm{m}$. B: Protein expressions of EPOr in carotid body of rat in Nx, IH and $\mathrm{CH}$ for 3 (D3), 14 (D14) and 28 days (D28). Data are presented in \% area with positive staining of EPOr immunoreactivity and are expressed as mean \pm SEM $(n=5$ for each group).

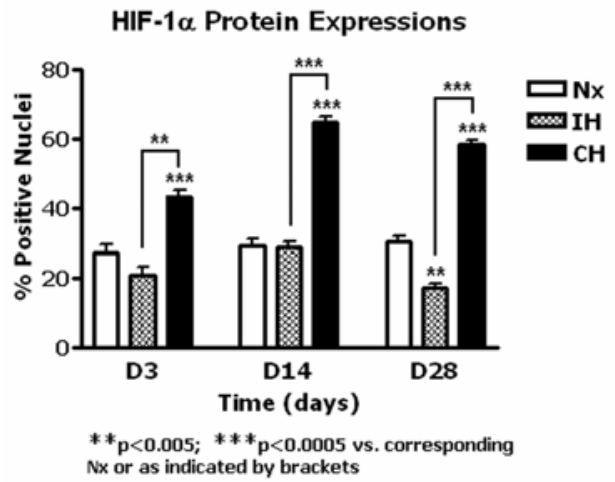

Figure 3. Protein expressions of HIF- $1 \alpha$ in the carotid body of rat in $\mathrm{Nx}, \mathrm{IH}$ and $\mathrm{CH}$ for 3 (D3), 14 (D14) and 28 days (D28). Data are presented in \% nuclei with positive staining of HIF-1 $\alpha$ immunoreactivity and are expressed as mean \pm SEM $(n=5$ for each group). Adapted and modified from Lam et al., Histol Histopathol (2008) 23:271-280. 
Physiological responses to hypoxia involve changes in gene expression that are mediated by the transcriptional activator HIF-1. As cellular oxygen concentration decreases, expression of HIF-1 $\alpha$ increases exponentially with the upregulation of various proteins such as EPO to exert the physiological effects (Wang et al. 1995). Along with the presence and upregulation of HIF- $1 \alpha$ in the nuclei of the CB in hypoxia, it has been demonstrated that EPO is indeed expressed in the CB glomus cells and its expression is increased in $\mathrm{CH}$ and $\mathrm{IH}$. Our study showed that the expression was more markedly increased in the $\mathrm{CH}$ than that of the IH groups throughout the time course, reaching a peak level at day 14. The differential expression of HIF- $1 \alpha$ in $\mathrm{CH}$ and $\mathrm{IH}$ might account for the expression profile of EPO under two different hypoxic conditions. Nonetheless, elevated EPO expression observed in the $\mathrm{CB}$ during $\mathrm{CH}$ and $\mathrm{IH}$ might contribute to the activation of the peripheral chemoreceptors in $\mathrm{CB}$ and the modulation of the $\mathrm{CB}$ chemotransduction in hypoxia.

EPO has been shown to modulate the ventilatory response to reduced oxygen supply despite its novel erythropoietic function in enhancing red blood cells production. Recent study has showed that exogenous EPO activates the peripheral chemoreceptor in $\mathrm{CH}$. The expression of EPOr in the medullary respiratory centre as well as in the CB chemosensitive type-I cells suggests that EPO could modulate the hypoxic ventilatory response at both the central and peripheral levels (Soliz et al. 2005; Soliz et al. 2007). In agreement, our results demonstrated that EPOr was expressed in the CB glomus cells and the expression was upregulated by both $\mathrm{CH}$ and $\mathrm{IH}$. Thus, the positive EPOr immunostaining was increased significantly in the 3-day $\mathrm{CH}$ group and was elevated considerably at peak levels by day 14 in both the IH and $\mathrm{CH}$ rats with a greater extent of elevation in the $\mathrm{CH}$ group. This expression profile is in conjunction to the HIF-1 $\alpha$ expression in the CB, although the EPOr expression was partially returned to the $\mathrm{Nx}$ level at day 28.

In summary, these results suggest an upregulation of the expression of EPO and its receptor in the $\mathrm{CB}$ glomic clusters in the $\mathrm{CH}$ and $\mathrm{IH}$ rats. The increased EPO expression in the $\mathrm{CB}$ may be mediated by the activation of HIF-1 pathway under hypoxic conditions. It is speculated that the increased expression of EPO and its receptor might be functionally related to the augmented $\mathrm{CB}$ hypoxic chemosensitivity and ventilatory chemoreflex under $\mathrm{CH}$ and $\mathrm{IH}$ conditions, which are relevant to the physiological acclimation to high altitude and the pathophysiological changes in patients with sleep-disordered breathing.

\section{ACKNOWLEDGEMENTS}


We thank Mr. W.B. Wong and Ms. K.M. Leung for their technical assistance. This work was supported by research grant (HKU 7510/06M) from the Research Grants Council, HKSAR and the University of Hong Kong.

\section{REFERENCES}

Bee, D., and Howard, P., 1993, The carotid body: a review of its anatomy, physiology and clinical importance, Monaldi Arch Chest Dis 48(1):48-53.

Chen, Y., Tipoe, G. L., Liong, E., Leung, S., Lam, S. Y., Iwase, R., Tjong, Y. W., and Fung, M. L., 2002, Chronic hypoxia enhances endothelin-1-induced intracellular calcium elevation in rat carotid body chemoreceptors and up-regulates ETA receptor expression, Pflugers Arch 443(4):565-73.

Fletcher, E. C., 2001, Invited review: Physiological consequences of intermittent hypoxia: systemic blood pressure, J Appl Physiol 90(4):1600-5.

Fung, M. L., 2003, Hypoxia-inducible factor-1: a molecular hint of physiological changes in the carotid body during long-term hypoxemia?, Curr Drug Targets Cardiovasc Haematol Disord $3(3): 254-9$.

Gonzalez, C., Almaraz, L., Obeso, A., and Rigual, R., 1994, Carotid body chemoreceptors: from natural stimuli to sensory discharges, Physiol Rev 74(4):829-98.

Heath, D., Edwards, C., and Harris, P., 1970, Post-mortem size and structure of the human carotid body, Thorax 25(2):129-40.

Jelkmann, W., 1992, Erythropoietin: structure, control of production, and function, Physiol Rev 72(2):449-89.

Kim, D. K., Natarajan, N., Prabhakar, N. R., and Kumar, G. K., 2004, Facilitation of dopamine and acetylcholine release by intermittent hypoxia in PC12 cells: involvement of calcium and reactive oxygen species, J Appl Physiol 96(3):1206-15; discussion 1196.

Lam, S. Y., Tipoe, G. L., Liong, E. C., and Fung, M. L., 2008, Differential expressions and roles of hypoxia-inducible factor1alpha, -2alpha and -3alpha in the rat carotid body during chronic and intermittent hypoxia, Histol Histopathol 23(3):27180.

Pagel, H., Engel, A., and Jelkmann, W., 1992, Erythropoietin induction by hypoxia. A comparison of in vitro and in vivo experiments, Adv Exp Med Biol 317:515-9.

Peng, Y. J., Rennison, J., and Prabhakar, N. R., 2004, Intermittent hypoxia augments carotid body and ventilatory response to hypoxia in neonatal rat pups, J Appl Physiol 97(5):2020-5. 
Prabhakar, N. R., 2001, Oxygen sensing during intermittent hypoxia: cellular and molecular mechanisms, J Appl Physiol 90(5):198694.

Soliz, J., Joseph, V., Soulage, C., Becskei, C., Vogel, J., Pequignot, J. M., Ogunshola, O., and Gassmann, M., 2005, Erythropoietin regulates hypoxic ventilation in mice by interacting with brainstem and carotid bodies, J Physiol 568(Pt 2):559-71.

Soliz, J., Soulage, C., Hermann, D. M., and Gassmann, M., 2007, Acute and chronic exposure to hypoxia alters ventilatory pattern but not minute ventilation of mice overexpressing erythropoietin, Am J Physiol Regul Integr Comp Physiol 293(4):R1702-10.

Tipoe, G. L., and Fung, M. L., 2003, Expression of HIF-1alpha, VEGF and VEGF receptors in the carotid body of chronically hypoxic rat, Respir Physiol Neurobiol 138(2-3):143-54.

Tramezzani, J. H., Morita, E., and Chiocchio, S. R., 1971, The carotid body as a neuroendocrine organ involved in control of erythropoiesis, Proc Natl Acad Sci U S A 68(1):52-5.

Wang, G. L., Jiang, B. H., Rue, E. A., and Semenza, G. L., 1995, Hypoxia-inducible factor 1 is a basic-helix-loop-helix-PAS heterodimer regulated by cellular O2 tension, Proc Natl Acad Sci U S A 92(12):5510-4. 